\title{
Überlebensverlängerung mit Abirateronacetat auch bei Patienten über 75 Jahren
}

— Von Abirateronacetat (Zytiga ${ }^{\circledR}$ ) profitieren einer aktuellen Auswertung zufolge auch Patienten im Alter über 75 Jahre [Mulders PF et al. EAU 2012; Abstract 127]. Abirateronacetat ist in Kombination mit Prednison/ Prednisolon für die Behandlung von Patienten mit metastasiertem kastrationsresistentem Prostatakarzinom (mCRPC) zugelassen, bei denen die Erkrankung während oder nach Docetaxel-haltiger Chemotherapie progredient verläuft. In der Post-hoc-Analyse der Zulassungsstudie, die im Rahmen des Kongresses der European Association of Urology (EAU) 2012 in Paris vorgestellt wurde, hatten Mulders et al. den primären Studie- nendpunkt - die Verlängerung des Gesamtüberlebens - bei Teilnehmern im Alter unter oder $\geq 75$ Jahre ausgewertet, weil die Diagnose Prostatakarzinom bei nahezu $90 \%$ der Betroffenen erst im Alter über 70 Jahre gestellt wird. Abirateronacetat plus Prednison/ Prednisolon (AAP) verlängerte in der Gesamtgruppe gegenüber Placebo plus Prednison/ Prednisolon (PP) das Gesamtüberleben um median 4,6 Monate (median 15,8 (AAP) vs. 11,2 (PP) Monate; $p<0,0001$ ) [Fizazi K et al. ECCO 2011; Abstract 7000]. Die signifikante Überlegenheit von AAP war unabhängig vom Alter der Patienten. In der Gruppe der unter 75-Jährigen betrug das Gesamtüberleben im
Median 15,9 (AAP) vs. 12,0 (PP) Monate ( $p=$ 0,0055), in der Gruppe der $\geq 75$-Jährigen 15,6 (AAP) vs. 9,3 (PP) Monate ( $p=0,0022$ ). Auch beim radiologischen progressionsfreien Überleben (AAP vs. PP: $<75$ Jahre $p \leq 0,0001$, $\geq 75$ Jahre $p=0,0019)$ und bei der PSA-Ansprechrate (AAP vs. PP: $<75$ Jahre $p \leq 0,0001$, $\geq 75$ Jahre $p \leq 0,0001$ ) profitierten beide Altersgruppen signifikant von Abirateronacetat; die Verträglichkeit war in beiden Gruppen vergleichbar [Mulders PF et al. EAU 2012; Abstract 127].

Nach Informationen von

Janssen-Cilag, Neuss

\section{Altershypogonadismus: Mit Testosteron Alltag besser meistern}

- „Vor dem Lebensende sind wir heute über Jahre auf medikamentöse und fremde Hilfe angewiesen. Die Gebrechlichkeit ist die neue Krankheit des Alters", so Prof. Bruno Lunenfeld, Vorsitzender der International Society for the Study of the Aging Male (ISSAM). Dass die Hilfsbedürftigkeit durch ein Testosterondefizit wie beim Altershypogonadismus begünstigt und durch Substitution verbessert wird, steht für ihn außer Frage. In Quer- und Längsschnittstudien seien niedrigere Testosteronwerte vergesellschaftet mit stärkerer Hilfsbedürftigkeit, vermindertem Gleichgewichtsgefühl oder vermehrten Stür- zen. Chronische Erkrankungen senken den Serumspiegel weiter ab. Bei höheren Serumwerten bleiben ältere Männer jedoch länger in körperlich guter Verfassung, können Alltagsaufgaben besser bewältigen, erleiden weniger Stürze, haben seltener einen Diabetes mellitus und scheinen selbst bei gesicherter Koronarkrankheit länger zu überleben. „Beim Altershypogonadismus liegen gesicherte Ergebnisse zum positiven Einfluss auf Muskel- und Knochenmasse sowie Libido und sexuelle Funktionen vor. Immer mehr Studien zeigen Verbesserungen beim Fettund Glukose-Metabolismus und verbesserte körperliche Funktionen bei eingeschränkter Mobilität", so Lunenfeld.

Bei der Applikationsform plädierte er für die transdermale Gabe, etwa Androtop ${ }^{\oplus} \mathrm{Gel}$ :„Anders als bei jungen sollte man mit Depot-Injektionen bei älteren Männern vorsichtig sein. Sie lassen das Testosteron und Hämoglobin sofort kräftig ansteigen." Auch hinsichtlich eines okkulten Prostatakarzinoms sind die Gelform oder orale Präparate günstiger, weil die Substitution sofort beendet werden kann, falls PSA oder Hämatokrit signifikant steigen.

Nach Informationen von Kade/Besins, Berlin

\section{IQWiG erkennt Zusatznutzen für Belatacept}

— Das Institut für Qualität und Wirtschaftlichkeit im Gesundheitswesen (IQWiG) hat sein Gutachten zur frühen Nutzenbewertung zu Belatacept $\left(\right.$ Nulojix $\left.^{\circledast}\right)$, ein selektiver T-Zell-Costimulationsblocker zur Prävention von Abstoßungsreaktionen bei erwachsenen nierentransplantierten Patienten, veröffentlicht. Es bestätigt für Belatacept einen geringen Zusatznutzen bei Erwachsenen mit Transplantaten von Spendern, die nach Standardkriterien ausgewählt waren. Für Patienten, die ein Transplantat eines Spenders nach erweiterten Kriterien erhalten hatten, sieht das IQWiG keinen Beleg für einen Zusatznutzen. Die Bewertung basiert auf den zwei randomisierten, multizentrischen, kontrollierten Phase-III-Studien BENEFIT und BENEFIT-EXT. In die BENEFITStudie waren Empfänger von Nieren von lebenden und verstorbenen Spendern mit nach Standard-Kriterien (SDC), in BENEFITEXT Empfänger von Transplantaten mit erweiterten Kriterien (ECD) eingeschlossen, die jeweils entweder mit Belatacept oder dem Immunsuppressivum Ciclosporin A ( $\mathrm{Cs} A$ ) behandelt wurden. In beiden Studien konnte gezeigt werden, dass Belatacept in Bezug auf die Endpunkte Patientenüberle- ben und Transplantatüberleben ebenso effektiv wie Ciclosporin A ist, und dass es die Nierenfunktion nach 12, 24 und 36 Monaten signifikant und nachhaltig besser aufrechterhält als unter Ciclosporin A [Vincenti F et al. ATC 2011; Abstract 227, Medina Pestana J et al. ATC 2011; Abstract 1088]. Das IQWiG-Gutachten hat empfehlenden Charakter. Einen Beschluss zur Nutzenbewertung für Belatacept trifft der G-BA voraussichtlich im Juli 2012.

Nach Informationen von

Bristol-Myers Squibb, München 